\title{
Seizures in Stroke Patients - A Prospective Study
}

\author{
A. Sethuram ${ }^{1}$, Y. Kingsly Jebasingh ${ }^{2}$ \\ ${ }^{1}$ Associate, Professor, Department of Neurology, Kanyakumari Government Medical College, Tamilnadu, ${ }^{2}$ Professor, \\ Department of Neurology, Kanyakumari Government Medical College, Tamilnadu, India
}

Corresponding author: Dr. Y. Kingsly Jebasingh, Professor, Department of Neurology, Kanyakumari Government Medical College, Tamilnadu, India

DOI: http://dx.doi.org/10.21276/ijcmsr.2019.4.4.6

How to cite this article: A. Sethuram, Y. Kingsly Jebasingh. Seizures in Stroke Patients - A Prospective Study. International Journal of Contemporary Medicine Surgery and Radiology. 2019;4(4):D26-D28.

\section{A B S T R A C T}

Introduction: Stroke, one of the major causes of epilepsy, accounts for up to $11 \%$ of epilepsy patients in western countries. American Stroke Association has recently reported that about one in six (15.3\%) stroke survivors experience seizures within about 3.4 years after suffering brain attack. Study aimed to assess and study the incidence and prevalence of seizures in stroke patients.

Material and Methods: 66 patients diagnosed with CVA above 18 years of age were included in this study. A detailed clinical history and meticulous neurological examination were done. Special neurological tests like CT scan brain, MRI (in selected patients) and EEG were also carried out in stroke patients.

Results: Incidence of seizures in the cerebrovascular accident patients is about $15 \%$. Seizures are common during early $48 \mathrm{hrs}$ of stroke (70\%). Partial seizure (motor type) is the most common $80 \%$. Incidence of Generalized Tonic-Clonic Seizures is $20 \%$. Cortical hemorrhagic lesions cause seizures more common than infarct (20\% VS $18 \%$ ). Response to Anti Epileptic Drugs is good $80 \%$. Recurrent seizures occurred in $20 \%$ of the cases.

Conclusion: Recognition of seizures and management in stroke patients will facilitate good recovery.

Keywords: Cerebrovascular Accident, Stroke, Seizure, Management

\section{INTRODUCTION}

Cerebrovascular disease (or) stroke rank foremost among all the CNS disorders. In the West, they represent as the third leading cause of death, with an overall prevalence rate of 800 per, 1,00,000 persons. It ranks after heart disease and cancer as the third commonest cause of death. ${ }^{1}$

Every year around 5 lakh cases of stroke, with about 1,75,000 fatalities from this cause. Since the active early detection and management of hypertension, there has been a great reduction in the frequency of stroke. So this world witnessed a gross reduction in cerebral infarct about $40 \%$ and haemorrhage $30 \%$ in between 1950-54. This reduction shared both sexes. There has been no change in the frequency of aneurysmal rupture. $^{2}$

Cerebral embolism is one of the important causes of stroke particularly in young patients. The incidence of cardiac embolism varies in several studies. It is as low as $3 \%$ in community studies and as high as 30\% in referral centers and $15 \%$ of patients with stroke. Intra cerebral haemorrhage accounts for approximately $10-15 \%$ of stroke. The commonest risk factor being hypertension. ${ }^{3}$

Reports on the frequency of seizures following stroke vary quite widely because of, differing stroke patient populations, sample sizes studies, varying follow up periods, definitions used for stroke and seizures, use of investigation such as CT and MRI and type of statistical analysis. In most studies to date the follow-up period was less than a few weeks, so the documentation of late-onset or recurring seizures is limited. ${ }^{4}$ Study aimed to assess and study the incidence and prevalence of seizures in stroke patients.

\section{MATERIAL AND METHODS}

All patients above 18 years of age admitted in Government Rajaji Hospital, Madurai with Cerebrovascular Accident are included in the study. Patients clinically diagnosed as CVA, above the 18 years of age were included. Exclusion criteria: Stroke and seizures due to toxemia of pregnancy are not included, venous stroke are not included. Stroke like presentation due to surgical causes like brain abscess, AVMs and CNS tumors are excluded (Primary and Secondary). CNS Infections and inflammatory vascular diseases manifesting as strokes are excluded. Strokes due to drug abuse [cocaine], poisoning and due to post-thrombolytic effects are excluded. Known seizure patients are also excluded.

A detailed clinical history and meticulous neurological examination were done. Examination of other systems was done and routine investigation like total count, differential count, HB, ESR, Chest X-ray, Electrocardiogram, Echocardiogram (selected cases), Trans-oesophageal echocardiogram (Selected cases only) were carried out. Special neurological tests like CT scan brain, MRI (in selected cases) and EEG were also carried out in stroke patients.

If the patient develops seizures, a clinical history of nature, 
type and pattern of seizures were analyzed. A specific question of secondary generalization if present (or) not was also asked. Inquiry about the worsening of the existing neurological deficit was also asked. Presence of complex partial attacks (CPC) and status epilepticus (SE) was specifically enquired. Inter-ictal electro-encephalography studies were carried out in these patients. The response to the anti-epileptic drugs was assessed and tabulated. Persistence of seizures even after anti-epileptic drugs were recorded. The nature and type of seizures and the stroke pattern have collaborated subsequent development of post-stroke epilepsy was enquired and the patient was followed. Any further worsening of the neurological status was noticed and recorded.

\section{RESULTS}

In this study 66 patients were included. The cerebrovascular disease may account for approximately $50 \%$ of new case of epilepsy in patients older than 65. In our study 2 patients who were above 75 years developed seizures following stroke. (Table 1)

Out of the total sixty-six patients 10 had seizures. 7 out of the 10 patients who developed seizures had seizures within 48 hours of the onset of stroke. 3 of the 10 patients developed delayed seizures. 1 patient developed late-onset seizures (after 2 weeks of stroke) but subsequently on imaging he was found to have multi-infarct state. None of the patients developed very late seizures (after 2 months). (Table 2) The patient just mentioned above who had multi-infarct state,

\begin{tabular}{|l|c|c|c|}
\hline \multirow{2}{*}{ Age group } & \multicolumn{2}{|c|}{ Sex } & \multirow{2}{*}{ Total } \\
\cline { 2 - 3 } & Male & Female & \\
\hline 18-30 years & 7 & 5 & 12 \\
\hline 31-40 years & 5 & 3 & 8 \\
\hline 41-50 years & 5 & 3 & 8 \\
\hline $51-60$ years & 12 & 10 & 22 \\
\hline 61-70 years & 5 & 2 & 7 \\
\hline 70 years and above & 6 & 3 & 9 \\
\hline Total & 40 & 26 & 66 \\
\hline \multicolumn{3}{|c|}{ Table-1: Age-wise distribution of patients with stroke is as } \\
follows \\
\hline
\end{tabular}

\begin{tabular}{|l|l|}
\hline Immediate (onset within 48 hours) & 7 \\
\hline Delayed (48 hours to 2 weeks) & 3 \\
\hline Above 2 weeks & 1 \\
\hline Very late (2 months and above) & 0 \\
\hline Recurrent seizures (post stroke seizures) & 2 \\
\hline \multicolumn{2}{|c|}{ Table-2: patients distribution according to clinical findings } \\
\hline
\end{tabular}

\begin{tabular}{|l|c|l|c|}
\hline $\begin{array}{l}\text { Imaging findings } \\
\text { brain ct scan }\end{array}$ & Nos & Type of seizures & Frequency \\
\hline Infarct & \multirow{2}{*}{49} & Partial & 7 \\
\cline { 3 - 4 } & & Generalized & 2 \\
\hline Haemorrhagic lesion & 5 & Partial & 1 \\
\cline { 3 - 4 } & & Generalized & 0 \\
\hline Total & 54 & & 10 \\
\hline
\end{tabular}

Table-3: Patients distribution according to imaging findings and type of seizures

\begin{tabular}{|l|c|}
\hline Infarct & Frequency \\
\hline Cortical infarct & 16 \\
\hline Corona radiata & 6 \\
\hline Capsuloganglionic infarct & 19 \\
\hline Lacunar infarct & 3 \\
\hline Thalamic infarct & 1 \\
\hline Brainstem infarct & 1 \\
\hline Cerebral infarct & 1 \\
\hline Multi-infarct & 2 \\
\hline Total & 49 \\
\hline \multicolumn{2}{|c|}{ Table-4: CT Findings } \\
\hline
\end{tabular}

\begin{tabular}{|l|c|}
\hline Haemorrhage & Frequency \\
\hline Putaminal Bleed & 3 \\
\hline Thalamic Bleed & 1 \\
\hline Intra Cerebral Bleed (Lobar) & 1 \\
\hline \multicolumn{2}{|c|}{ Table-5: MRI Findings } \\
\hline
\end{tabular}

\begin{tabular}{|l|c|c|}
\hline Stroke-Territory & $\begin{array}{c}\text { No. of } \\
\text { cases }\end{array}$ & $\begin{array}{c}\text { With } \\
\text { seizures }\end{array}$ \\
\hline Anterior (carotid) & 56 & 9 \\
\hline Posterior (vertebra-basilar territory) & 10 & 1 \\
\hline Total & 66 & 10 \\
\hline \multicolumn{2}{|c|}{ Table-6: Stroke-Territory } \\
\hline
\end{tabular}

used to get recurrent seizures (post-stroke epilepsy). 1 patient who had occipital infarct also used to get recurrent seizures, inspite of antiepileptic drugs. Rest of the other patient did not develop a recurrent seizure. (Table 3) On analyzing the distribution of imaging findings and type of seizures, out of the 49 patients with infarct 7 had partial seizures. Of the seven with partial seizures 4 had partial seizures alone, 3 had for partial seizures with secondary generalization and 2 had generalized tonic-clonic seizures. Of the 5 haemorrhagic lesions in CT brain 1 patient developed partial seizures. This shows that haemorrhagic lesion of the cortex is prone to develop seizures than infarcts. (Table 4 and 5) Of these one patient with ICH developed seizures. In the infarct group, out of 49 patients 9 developed seizures. In our study of 66 patients; strokes involving the anterior (carotid) circulation is 56 patients and strokes involving the posterior circulation is 10 . Of the ten patients with post-stroke seizures; 9 cases have had stroke involving anterior circulation, and 1 case had a stroke involving posterior circulation. (Table 6)

\section{DISCUSSION}

Stroke is the leading cause of seizures in the older population, especially beyond 60 years of age. Cerebral vascular disease (infarction (or) haemorrhage) accounts for 14 to 54\% of diagnosed cases of seizures in older patients according to the most recent studies. A similar trend in the age-specific incidence of seizures was seen in the community based study in Rochester Minnesota by Hauser. This observation fits and corresponds well with our observation. ${ }^{5}$

Partial seizures predominate after cerebral infarction and haemorrhage by a study conducted by Fraught et al. ${ }^{6}$ Similar to our study, partial seizures of the simple motor type which 
is the most common type in our study correlated very well in the findings of other studies. 2 of our cases had GTCS. According to other studies tonic-clonic seizures are also common but it is probable, that in many the focal onset is un-witnessed or not appreciated.

Status epilepticus (SE) is rarely reported following stroke. None of our patients had SE. (both convulsive and nonconvulsive SE).Lobar haemorrhages are commonly associated with SE than other types of intracerebral haemorrahge. As already mentioned CVA is one of the causes of $\mathrm{SE}$ in older patients. In addition, the mortality of SE increases with advancing age beyond 60 years and duration of seizures. Hence early recognization and management of seizures is very important. Important cause of SE after stroke are anticonvulsant withdrawal and non-compliance.

In an Indian study by Dhanuka $77 \%$ of post-stroke seizures occurred as early-onset seizures with $2 / 3$ being immediate post-stroke seizures. ${ }^{7}$ In comparison, our study shows $70 \%$ of post-stroke seizures occur as early-onset seizures, and 30\% delayed seizures.

Dhanuka study demonstrates that recurrent seizures are less with early-onset seizures; this correlates well with our study too. $^{7}$

Status epilepticus following stroke is uncommon $(<10 \%)$. In correlation with our study none of the patients developed status epilepticus (SE).

The cortical lesion is a risk factor for post-stroke seizures, more with larger lesions that involve multiple lobes of the cortex. Lobar haemorrhage is considered to be the most epileptogenic in locations. Lobar haemorrhage accounts for $54 \%$ and basal ganglia bleed in $19 \%$, in a series of 123 patients studies by Faught. ${ }^{6}$ In our study out of the 5 haemorrhagic strokes 1 patient developed seizures.

According to a study by Richardson and Dodge, Arboix et al- the most consistent risk factor for seizures at the onset is following cerebral infarction or haemorrhage with cortical involvement. With respect to intracerebral haemorrhage, lobar haemorrhage carriesa higher risk of seizures compared to those located sub-cortically. ${ }^{8,9}$ This result correlate very well with our observation.

All of our patients with post-stroke seizures, were treated with oral Phenytoin; 8 of our patients responded very well to phenytoin alone, amounting to eighty percent of response to anti-epileptic drugs. Two of our patients still had poststroke seizures. Of these 2 patients one was diagnosed to have multi-infarct state and continue to have seizures. None of our patients was intolerant, no adverse reactions developed to the administration of Phenytoin. Finally in pre-existing neurological deficits due to stroke, the neurological deficit may worsen after a prolonged seizure, this indicates the need for prompt seizure control.

\section{CONCLUSION}

In conclusion the incidence of seizures in stroke is about 15\%. Partial seizures are the mostcommon seizure type. Complex partial seizure and status epilepticus were not observed. Response to conventional antiepileptic drugs is good. Recognization and management of seizures in stroke patients will facilitate recovery and decrease disability.

\section{REFERENCES}

1. Thomas Truelsen, Stephen Begg, Colin Mathers. The global burden of cerebrovascular disease [Internet]. Who.int. 2002 [cited 8 August 2019]. Available from: https://www.who.int/healthinfo/statistics/bod_ cerebrovasculardiseasestroke.pdf

2. Pandian JD, Sudhan P. Stroke epidemiology and stroke care services in India. J Stroke. 2013;15(3):128-134.

3. Smajlović D. Strokes in young adults: epidemiology and prevention. Vasc Health Risk Manag. 2015;11:157-164.

4. Lackland DT, Roccella EJ, Deutsch AF, et al. Factors influencing the decline in stroke mortality: a statement from the American Heart Association/American Stroke Association. Stroke. 2014;45(1):315-353.

5. Hauser W, Annegers J, Kurland L. Prevalence of Epilepsy in Rochester, Minnesota: 1940-1980. Epilepsia. 1991;32(4):429-445.

6. E. Faught, D. Peters, A. Bartolucci, L. Moore, and P. C. Miller. Seizures after primary intracerebral hemorrhage. Neurology 1989;39(8):1089-1093.

7. Dhanuka A K, Misra U K, Kalita J. Seizures after stroke: a prospective clinical study. Neurol India 2001;49:33.

8. Richardson EP, Jr, Dodge PR. Epilepsy in cerebral vascular disease: a study of the incidence and nature of seizures in 104 consecutive autopsy-proven cases of cerebral infarction and hemorrhage. Epilepsia. 1954;3:49-74.

9. Arboix A, Garcia-Eroles L, Massons JB, Oliveres M, Comes E. Predictive factors of early seizures after acute cerebrovascular disease. Stroke. 1997;28:1590-1594.

Source of Support: Nil; Conflict of Interest: None

Submitted: 08-08-2019; Accepted: 29-08-2019; Published online: 14-10-2019 